University of Nebraska - Lincoln

DigitalCommons@University of Nebraska - Lincoln

Faculty Publications: Materials Research

Science and Engineering Center

Materials Research Science and Engineering

Center

2003

\title{
Bistable memory effect in chromium oxide junctions
}

\author{
Andrei Sokolov \\ University of Nebraska-Lincoln, sokolov@unl.edu \\ C.-S. Yang \\ University of Nebraska - Lincoln \\ E. Ovtchenkov \\ University of Nebraska - Lincoln \\ L. Yuan \\ University of Nebraska - Lincoln \\ Sy-Hwang Liou \\ University of Nebraska-Lincoln, sliou@unl.edu
}

See next page for additional authors

Follow this and additional works at: https://digitalcommons.unl.edu/mrsecfacpubs

Part of the Materials Science and Engineering Commons

Sokolov, Andrei; Yang, C.-S.; Ovtchenkov, E.; Yuan, L.; Liou, Sy-Hwang; and Doudin, Bernard, "Bistable memory effect in chromium oxide junctions" (2003). Faculty Publications: Materials Research Science and Engineering Center. 83.

https://digitalcommons.unl.edu/mrsecfacpubs/83

This Article is brought to you for free and open access by the Materials Research Science and Engineering Center at DigitalCommons@University of Nebraska - Lincoln. It has been accepted for inclusion in Faculty Publications: Materials Research Science and Engineering Center by an authorized administrator of DigitalCommons@University of Nebraska - Lincoln. 


\section{Authors}

Andrei Sokolov, C.-S. Yang, E. Ovtchenkov, L. Yuan, Sy-Hwang Liou, and Bernard Doudin 


\title{
Bistable memory effect in chromium oxide junctions
}

\author{
A. Sokolov, C.-S.Yang, E. Ovtchenkov, L. Yuan, S.-H. Liou, B. Doudin \\ University of Nebraska Lincoln, Department of Physics and Astronomy, 115 Brace Laboratory, Lincoln \\ NE 68588-0111
}

\begin{abstract}
Magnetotransport properties of granular $\mathrm{CrO}_{2} / \mathrm{Cr}_{2} \mathrm{O}_{3}$ films made of $\mathrm{CrO}_{2}$ crystals covered by 1- $2 \mathrm{~nm}$ native insulating $\mathrm{Cr}_{2} \mathrm{O}_{3}$ are presented. Electrical properties of a limited number of grains measured in series and parallel (10 to 15 grains) reveal intergrain tunneling characteristics. At lowest temperatures, a well pronounced zero bias anomaly indicates that impurities in the junctions block the electronic flow.

Hysteresis in the IV curves are observed at intermediate temperatures on zero-field cooled samples. Changing the polarity of a short excitation pulse (100ns) of amplitude smaller than $1 \mathrm{~V}$ triggers a change in the zero-bias resistance by $10-50 \%$. These states are stable and well reproducible in the temperature interval ranging from $100 \mathrm{~K}$ to $250 \mathrm{~K}$. Applying an external magnetic field cancels the IV hysteresis. The resistance of the devices in the $\mathrm{k} \Omega$ range, the potential high-speed for writing and reading the resistance sate, make these systems interesting candidates for magnetic non-volatile memories.
\end{abstract}

\section{INTRODUCTION}

The integration of magnetic materials in electronic components has become of increasing importance since the boost of research and development efforts initiated by the discovery of giant magnetoresistance effects. This new area of "spintronics" [1] opens the possibility to use magnetic configuration control as an extra degree of freedom for tuning the electric transport properties. The materials with high degree of spin polarization are the most desired for potential application in metal/insulator/metal devices [2], and magnetic multilayer geometries [3, 4].

Chromium dioxide $\left(\mathrm{CrO}_{2}\right)$ is among the best candidates for half-metallic ferromagnetic systems. Evidence of large spin polarization of conduction electrons has been observed using several methods [5-7]. Magnetoresistance (MR) measurements have been reported in polycrystalline films [8-10], epitaxial films [10- 12] and powder compacts of $\mathrm{CrO}_{2}$ [13]. Magnetoresistance values reaching $50 \%$ have been observed on $\mathrm{CrO}_{2} / \mathrm{Cr}_{2} \mathrm{O}_{3}$ composites at low temperature [13]. The results were explained in terms of intergrain tunneling, and the large magnetoresistance (MR) values were attributed to the high degree of spin polarization existing in $\mathrm{CrO}_{2}$. In spite of relatively high Tc, of about $390 \mathrm{~K}$, the MR value decays rapidly with increasing temperature and disappointed few percent remains at ambient temperature. A possible explanation of such a behavior is given in terms of Coulomb blockade effects [14].

Another attractive feature of spin-electronic devices is the possibility of current induced magnetization reversal. Injection of spin-polarized electric current can modify the magnetic states of a small ferromagnet. This spin-transfer effect was theoretically predicted by Berger [15] and Slonczewski [16] and there are several experiments giving evidence of modification of the magnetization configurations in nano-magnetic materials induced by an electric current [17-22]. 

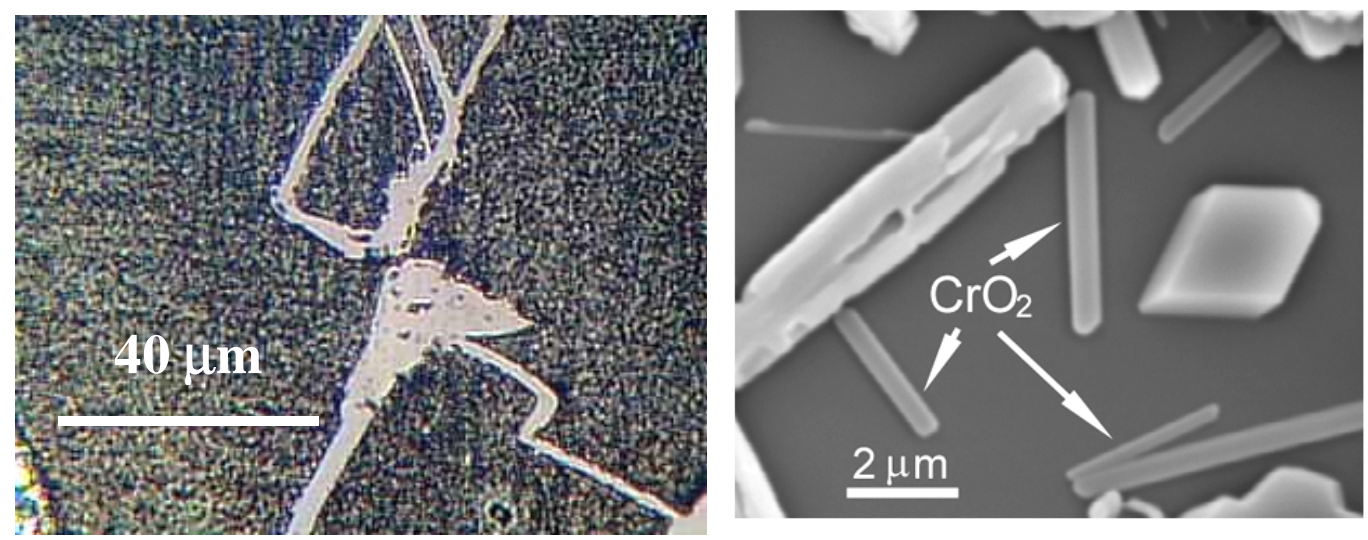

Fig.1 Optical micrograph (left) and SEM picture (right) of the chromium oxide thin films. Left picture show the geometry and typical size of mechanically scribed samples. Right picture shows a low-density film exhibiting typical sizes and shapes of the $\mathrm{CrO}_{2}$ crystals.

In this paper we report the results of our study of compact powder $\mathrm{CrO}_{2}$ in wide temperature interval of $1.5-275 \mathrm{~K}$. Large bias current were applied in order to investigate the possibility of current-induced switching.

\section{SAMPLE PREPARATION}

Polycrystalline $\mathrm{CrO}_{2}$ films were made by $\mathrm{RF}$ sputtering of a $\mathrm{CrO}_{3} / \mathrm{Cr}_{2} \mathrm{O}_{5}$ target (obtained by sintering a pressed $\mathrm{CrO}_{3}$ powder at $150^{\circ} \mathrm{C}$ ) onto $\mathrm{LaAlO}_{3}$ substrates and annealing in a high pressure cell. The sputtering was performed under of 10 mtorr argon and 2 mtorr oxygen pressure. Thickness of $\mathrm{CrO}_{3}$ films ranged between $0.1 \mu \mathrm{m}$ and $1 \mu \mathrm{m}$. Treatment in about 100 atms of oxygen pressure at $390^{\circ} \mathrm{C}$ for 10 hours lead to stable $\mathrm{CrO}_{2}$ phase, as confirmed by X-ray diffraction diagrams, with no detectable trace of $\mathrm{Cr}_{2} \mathrm{O}_{3}$. The thinnest annealed films showed a grain density of about 10 crystals every $100 \mu \mathrm{m}^{2}$ area (Fig.1). The grains were generally elongated shapes, typically $5 \mu \mathrm{m}$ long and $0.5 \mu \mathrm{m}$ wide. Subsequent mechanical scribing allowed us to limit the sample to a limited number of crystals [14] (10-15) in series and in parallel (Fig. 1). Electrical contacts separated by typically 200 microns. Measurements were performed by $\mathrm{DC}$ and $\mathrm{AC}$ techniques in a 4-probe geometry.

\section{RESULTS AND DISCUSSION}

Photoemission experiments revealed that the $\mathrm{CrO}_{2}$ crystals are covered by $1-2 \mathrm{~nm}$ of insulating $\mathrm{Cr}_{2} \mathrm{O}_{3}$ [23]. This thermodynamically more stable oxide phase plays the role of the native tunnel barrier between two adjacent crystallites. The temperature-dependent magnetoresistance properties for thick films (Fig. 2) are in qualitative agreement with previously reported results by others (for example [13]). The insert shows $\mathrm{R}(\mathrm{H})$ curves, normalized to $\mathrm{R}$ ( $\mathrm{H}$ $=1 \mathrm{~T}$ ). Increasing the applied field up to 9T shows continuous drop of resistance without visible saturation, which is consistent with scattering by magnetic impurities in the $\mathrm{CrO}_{2}$ crystals [24]. Systematic studies of the IV curves and bias-dependent magnetoresistance properties, combined with photoemission studies [14], showed that the low-temperature magnetoresistance is 


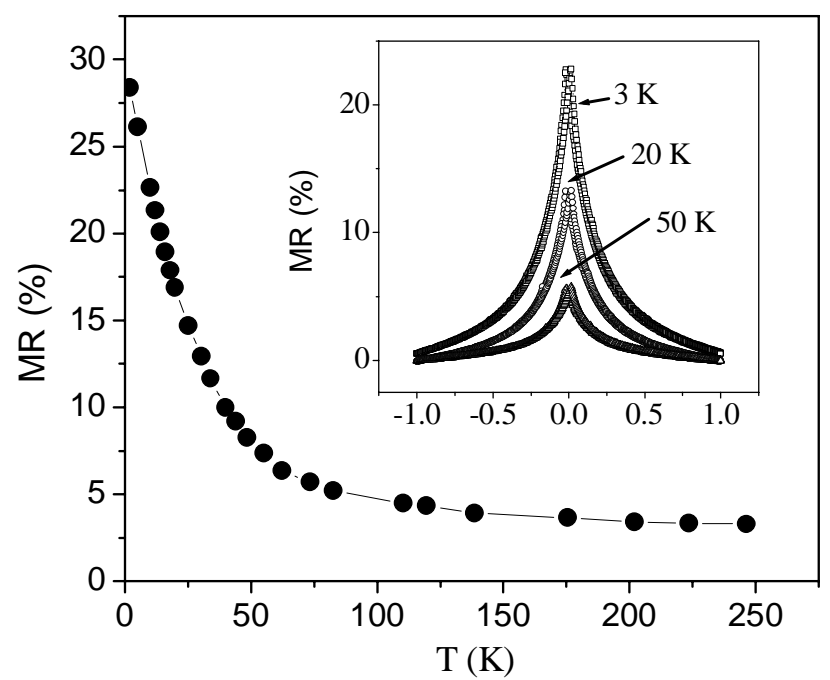

Fig.2 Temperature dependence of magnoresistance $(\mathrm{R}(\mathrm{H})-\mathrm{R}(\mathrm{H}=1 \mathrm{~T}) / \mathrm{R}(\mathrm{H}=1 \mathrm{~T})$, ıormalized to $\mathrm{R}$ at $\mathrm{H}=1 \mathrm{~T}$. Curves at several temperatures are depicted in the insert.

enhanced by Coulomb blockade effects though co-tunneling leakage current [25]. Inclusions in the barrier or near the $\mathrm{CrO}_{2} / \mathrm{Cr}_{2} \mathrm{O}_{3}$ interface play the role of Coulomb island, of several meV blocking energy.

The related zero-bias anomaly at lowest temperature is commonly attributed to blockade of current by impurities in the barrier. Comparisons of the zero-bias anomaly voltage width with

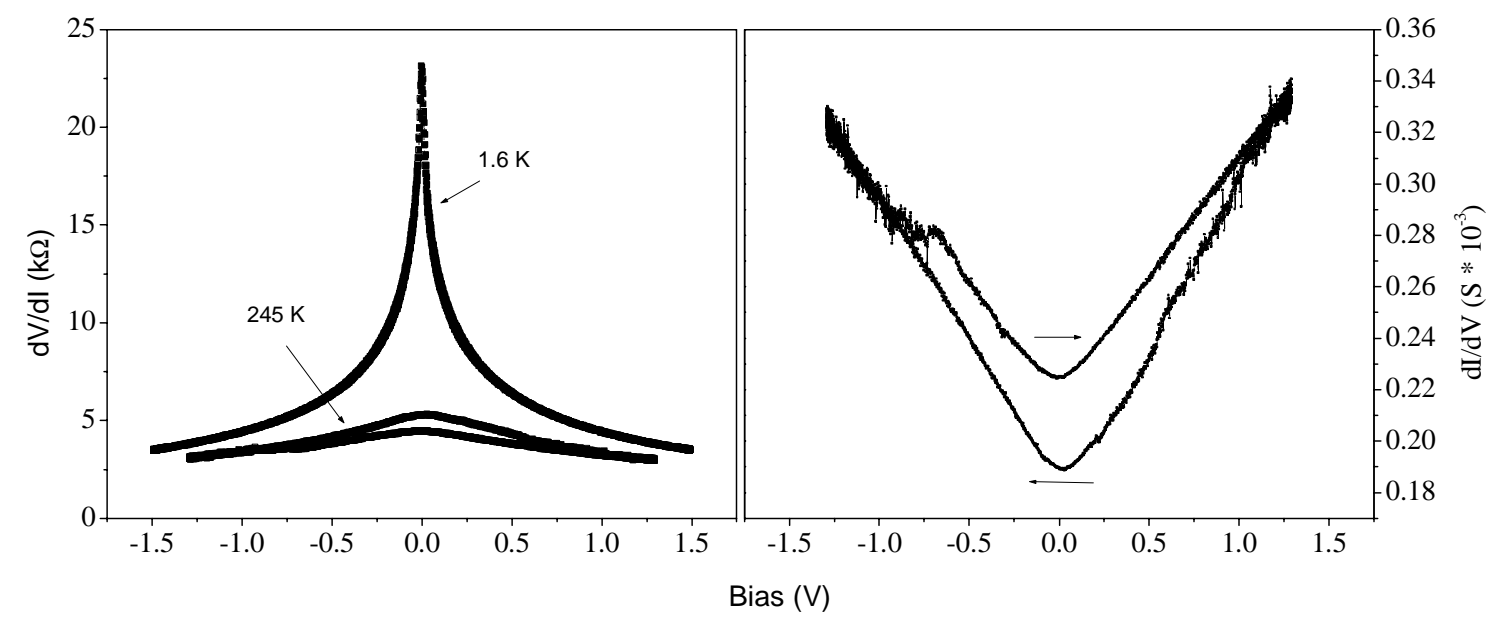

Fig. 3. Left: Differential resistance $\mathrm{dV} / \mathrm{dI}$ versus applied $\mathrm{DC}$ bias at different temperatures. A zero bias anomaly is pronounced at low temperatures and hysteresis in the curve is fount at higher temperatures. Right: Differential conductance dI/dV curve, measured at $245 \mathrm{~K}$, shows indications of a threshold behavior for the hysteresis 
observations by others [26], allows us to estimate that around 10 junctions are measured in series.

Sequential tunneling dominates the electronic transport in intergrain junctions at temperatures higher than several tens of K [14]. The resistance versus voltage (current) curves show non-reproducible characteristics. More precisely, hysteretic behavior in these curves is observed in temperatures ranging from $100 \mathrm{~K}$ to $250 \mathrm{~K}$, being occasionally as low as $40 \mathrm{~K}$ and as high as 300 K. (Fig. 3, Fig.4). The effect disappears after applying a magnetic field typically larger than $1 \mathrm{kOe}$, and the resistance value stabilizes within the envelope of the hysteretic curve (Fig. 4). Heating effects when biasing the sample with large voltage (current) values are limited, because the normal IV curve observed after applying a magnetic field becomes hysteretic again after heating the sample to more than $400 \mathrm{~K}$, and cooling under zero-field. These observations indicate that the hysteresis in the IV curves has a magnetic origin, related to the magnetic configuration at the junctions interfaces. The amplitude $\Delta$ of the bistability was investigated as a function of the maximum voltage, and a threshold-type behavior was found. We also tested the speed of the process, being able to keep the same $\Delta$ value when reducing the pulse duration down $100 \mathrm{~ns}$ (Fig. 5). Note that one state is less reproducible than the other when applying a voltage pulse. This observation at $245 \mathrm{~K}$ is related to the observation of a "fast" time decay of the high conductance state, showing a relaxation behavior of typical $10^{4}$ s time scale at $275 \mathrm{~K}$. Our experimental set-up essentially limits the pulse time, where amplifying electronics external to the cryostat are connected by significant cabling impedance, absorbing half of the charge correspondigng to the $100 \mathrm{~ns}$ pulse.

Although the origin of this bi-stable memory effect is yet to be understood, the relevance of a model of current induced magnetization switching is discussed. The existence of threshold potential and the effect of a magnetic field could be ascribed to spin transfer on the

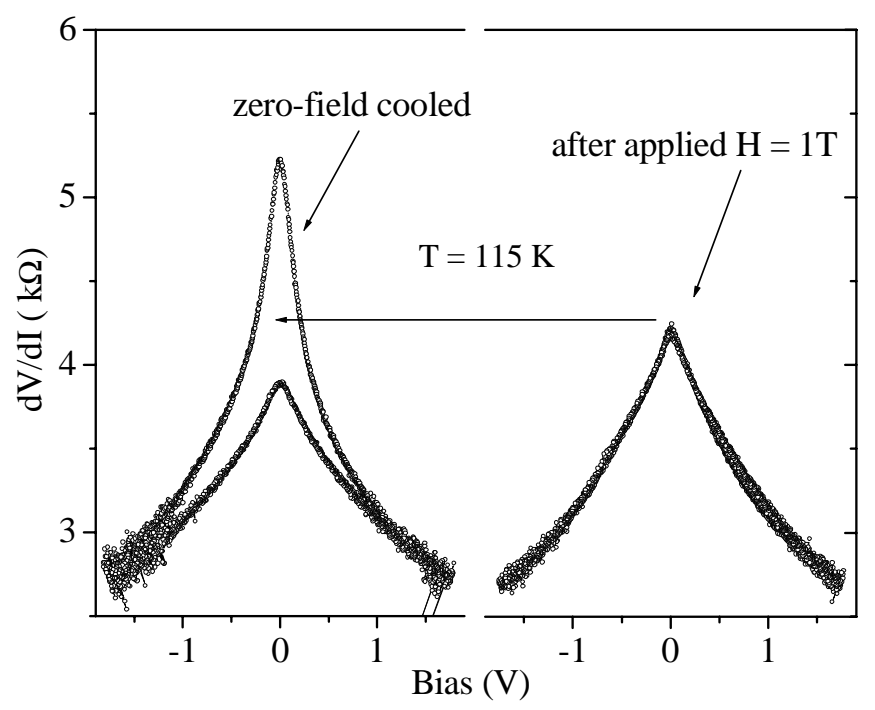

Fig. 4. Differential resistance as a function of current bias for 10-15 chromium junctions in series at $115 \mathrm{~K}$. Left: the resistance value after a positive bias is significantly smaller than after a negative bias. Right; the effect disappears after applying a magnetic field. 
magnetization direction of a small magnetic impurity, embedded in the barrier between two crystal with initial antiparallel alignment. We suppose a system made of two large magnets corresponding to the two neighbor $\mathrm{CrO}_{2}$ crystals, and a nanomagnet embedded in the barrier, causing the observed Coulomb blockade at low temperatures. The nanomagnet orientation is being flipped parallel to the magnetization of the source of spin-polarized electrons. Reversing the current direction will the change the magnetic orientation of the nanomagnet. IN such a device made of two tunnel junctions is series, with one junction in the parallel configuration (source) and the other one anti-parallel(drain). Reversing the current exchange thw two configurations. If one resistance is intrinsically very different form the other (for example if the nanomagnet is not equidistant from the two electrodes), the current-induced change of resistance $\Delta$ can reach the tunnel magnetoresistance value. An applied external field would realign the magnetizations at the interfaces and the asymmetry in the system vanishes. The existence of antiparallel magnetic alignment, which cannot be retrieved after applying an external magnetic field, is reminiscent of findings on magnetic multilayers used for CPP-MR investigations [27]. In other words, the antiparallel alignment of two neighbor crystals is maximized when the samples are in a virgin random magnetic state. An essential limitation for current-induced magnetization switching on tunnel junctions systems is the necessity to apply very large current densities. Investigations on Co nanomagnets showed critical current densities values larger than $10^{7} \mathrm{~A} / \mathrm{cm}^{2}$ $[18,22]$ for area typically $10^{3}-10^{4} \mathrm{~nm}^{2}$, in agreement with theoretical predictions. If we consider that a current of $1 \mu \mathrm{A}$ is forced though an island of a few $\mathrm{nm}$ in diameter, current densities of $10^{7}$ $\mathrm{A} / \mathrm{cm}^{2}$ can be reached. It corresponds to our observation, where several tens of $\mu \mathrm{A}$ were necessary to observe bistability. Such effects were only found on samples with thin $\mathrm{Cr}_{2} \mathrm{O}_{3}$ barriers, of a few $\mathrm{k} \Omega$ estimate of single junction resistance, and therefore classified in the strong

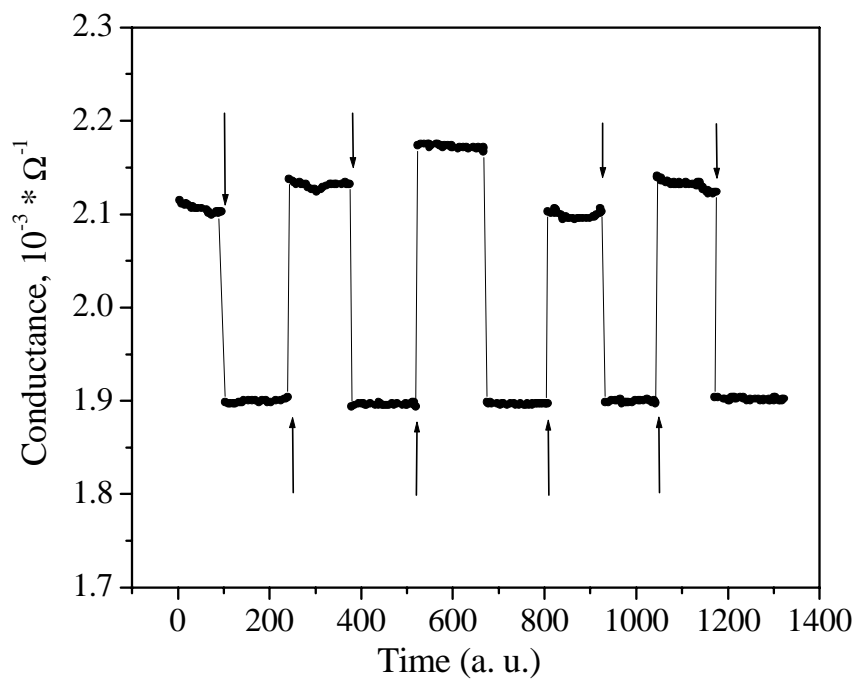

Fig. 5. Zero-bias conductance after applying a $100 \mathrm{~ns}$ pulse of $1 \mathrm{~V}$ amplitude at time values orresponding to the arrows. The temperature is $245 \mathrm{~K}$. The high-conductance state, lacking eproducibility after applying a voltage pulse, shows strong relaxation effects at higher temperatures. 
tunneling regime [14].

\section{CONCLUSIONS}

Chromium oxide junctions, showing Coulomb blockade effects at low temperatures, exhibit bistable resistance values at zero bias after applying a voltage of around $1 \mathrm{~V}$, involving a current of a few tens of $\mu \mathrm{A}$. The typical resistance values of several $\mathrm{k} \Omega$ of our samples make them of interest for applications as non-volatile memory devices. A model of current-induced switching is used to explain the results, using a geometry involving a small particle trapped between two large magnets with antiparallel alignments. We show that current densities approaching those predicted and observed for metallic systems can be realized in strong tunneling regime involving very low area junctions.

\section{REFERENCES}

[1] G.A. Prinz, Science 282, 1660 (1998).

[2] W. J. Gallagher et al., J. Appl. Phys. 81, 3741 (1997).

[3] K. Bussman, G. A. Prinz, S. F. Cheng and D. Wang, Appl. Phys. Lett. 75, 2476 (1999).

[4] N. Kikuchi et al., J. Appl. Phys. 90, 6548 (2001).

[5] Yu. S. Dedkov et al., Appl. Phys. Lett. 80, 4181 (2002).

[6] R. J. Soulen Jr. et al., Science 282, 85 (1998).

[7] Y. Ji et al., Phys. Rev. Lett. 86, 5585 (2001)

[8] A. Gupta, X. W. Li and Gang Xiao, J. App. Phys., 87, 6073, (2000).

[9] S. M. Watts, et al., Phys. Rev. B, 61, 9621, (2000).

10] Katsuhiko Suzuki and P.M. Tedrow, Appl. Phys. Lett., 74, 428 (1999).

[11] S. J. Liu et al., Appl. Phys. Lett., 80, 4202, (2002).

[12] P. A. Stampe, R. J. Kennedy, S. M. Watts and S. von Molnár, J. Appl. Phys., 89, 7696, (2001).

[13] J. M. D. Coey, A. E. Berkowitz, L1. Balcells, and F. F. Putris, Phys. Rev. Lett., 80, 3815 (1998).

[14] A. Sokolov et al., Europhys. Lett 58, 448 (2002).

[15] L. Berger, Phys. Rev. B 54, 9353-9358 (1996).

[16] J. Slonczewski, J. Magn. Magn. Mater. 159, L1 (1996).

[17] E.B. Myers et al., Science 285, 867 (1999).

[18] F. J. Albert, J. A. Katine, R. A. Buhrman and D. C. Ralph, Appl. Phys. Lett. 77, 3809 (2000).

[19] J. Z. Sun, J. Magn. Magn. Mater. 202, 157 (1999).

[20] J.E. Wegrowe et al., Europhys. Lett. 45, 626 (1999).

[21] M Tsoi et al., Phys. Rev. Lett. 80, 4281 (1998).

[22] J. Grollier et al., Appl. Phys. Lett. 78, 3663 -3665 (2001).

[23] Ruihua Cheng et al., Appl. Phys. Lett. 79, 3122 (2001).

[24] L. Yuan et al., submitted to J. Appl. Phys.

[25] S. Takahashi and S. Maekawa, Phys. Rev. Lett. 80, 1758 (1998).

[26] J. M. Rowell and L. Y. L. Shen, Phys. Rev. Lett. 17, 15 (1966)

[27] J. A. Borchers et al., Phys. Rev. Lett 82, 2796 (1999). 